\title{
Matrix Factorization Techniques for Context-Aware Collaborative Filtering Recommender Systems: A Survey
}

\author{
Mohamed Hussein Abdi $^{1}$, George Onyango Okeyo ${ }^{2}$ \& Ronald Waweru Mwangi ${ }^{2}$ \\ ${ }^{1}$ Department of Information \& Communication Technology, Kenya Medical Research Institute, Kenya \\ ${ }^{2}$ Department of Computing, School of Computing \& Information Technology, Jomo Kenyatta University of \\ Agriculture \& Technology, Kenya \\ Correspondence: Mohamed Hussein Abdi, Kenya Medical Research Institute P.O. Box 231-00610 Nairobi, \\ Kenya. Tel: 254-722-977-728 E-mail: mhabdi@gmail.com
}

Received: January 15, 2018

Accepted: January 24, 2018

Online Published: March 16, 2018

doi:10.5539/cis.v11n2p1

URL: http://dx.doi.org/10.5539/cis.v11n2p1

\begin{abstract}
Collaborative Filtering Recommender Systems predict user preferences for online information, products or services by learning from past user-item relationships. A predominant approach to Collaborative Filtering is Neighborhood-based, where a user-item preference rating is computed from ratings of similar items and/or users. This approach encounters data sparsity and scalability limitations as the volume of accessible information and the active users continue to grow leading to performance degradation, poor quality recommendations and inaccurate predictions. Despite these drawbacks, the problem of information overload has led to great interests in personalization techniques. The incorporation of context information and Matrix and Tensor Factorization techniques have proved to be a promising solution to some of these challenges. We conducted a focused review of literature in the areas of Context-aware Recommender Systems utilizing Matrix Factorization approaches. This survey paper presents a detailed literature review of Context-aware Recommender Systems and approaches to improving performance for large scale datasets and the impact of incorporating contextual information on the quality and accuracy of the recommendation. The results of this survey can be used as a basic reference for improving and optimizing existing Context-aware Collaborative Filtering based Recommender Systems. The main contribution of this paper is a survey of Matrix Factorization techniques for Context-aware Collaborative Filtering Recommender Systems.
\end{abstract}

Keywords: collaborative filtering, context-aware, matrix factorization, performance improvement, accuracy of predictions, quality of recommendations

\section{Introduction}

Recommender Systems are a class of web applications that assist users to tame the problem of information overload by providing personalized recommendations on various types of information, products and services. The existing Recommender Systems applications can be classified into specific domains like entertainment, e-commerce, e-learning and service recommendations in travel, expert consultation and match making (Rajabpour, Bardsiri, Mohammadighavam, \& Molaei, 2014). The various techniques used in generating recommendations suffers sparsity and scalability problem as the volume of accessible information and users continue to grow leading to accuracy and quality reduction. However, the popularity of the approach has drawn a great deal of research towards improving the prediction accuracy and the quality the recommendations. The interest in this area still remains high due to growing demand on practical applications, which are able to provide personalized recommendations and deal with information overload effectively (L. Sharma \& Gera, 2013). This is due to the importance of personalization techniques which does not only aim to provide tailored information to customers based on their preferences, restrictions or tastes but also increase profits of commercial systems (Gao, Liu, \& Wu, 2010).

These growing demands pose some key challenges to Recommender Systems and to deal with these problems many advanced techniques like Content-boosted Collaborative Filtering (Melville, Mooney, \& Nagarajan, 2002), Content-boosted Matrix Factorization (Nguyen \& Zhu, 2013), and the incorporation of contextual information (Adomavicius \& Mobasher, 2011; Adomavicius, Sankaranarayanan, Sen, \& Tuzhilin, 2005) have been geared towards improving the accuracy of recommended predictions. Context-aware Recommender Systems (CARS) 
approaches have been shown to provide more accurate predictions and relevant recommendations (Baltrunas, 2008; Karatzoglou, Amatriain, Baltrunas, \& Oliver, 2010).

Two major approaches are used in Collaborative Filtering: the Neighborhood-based (Breese, Heckerman, \& Kadie, 1998) and the Matrix Factorization (Koren, Bell, \& Volinsky, 2009). The Neighborhood approach relies on the preferences of the user's neighbor, that is, other users with similar preferences to estimate the user preferences. Matrix Factorization in its basic form, characterizes both items and users by vectors of factors. These factors are inferred from patterns of item ratings (Koren \& Bell, 2015). Matrix Factorization represents the relation between users and items through a set of latent factors also referred to as features. It forms two low rank matrices, each representing the relation between users (or items) and the set latent features. The multiplication of these two matrices allows estimating users' future preferences (Chertov, Brun, Boyer, \& Aleksandrova, 2015).

In this paper we explore the various Matrix Factorization approaches that have been studied in research aimed at improving performance for large scale datasets and the impact of incorporating contextual information.

\subsection{Evaluation Metrics}

There is no common evaluation framework that can be applied to all Recommender Systems. However, a variety of measures exists to evaluate their properties. Three types of metrics to measure the quality of an algorithm are commonly in use: prediction, classification and rank accuracy (Herlocker, Konstan, Terveen, \& Riedl, 2004). Prediction accuracy measures the difference between the rating the system predicts and the real rating. The most popular of this kind of metric is the Mean Absolute Error (MAE). Additional related metrics in use are the Mean Squared Error (MSE), Root Mean Squared Error (RMSE) or normalized Mean Absolute Error. The classification accuracy measures how well the system differentiates good items from bad ones. Examples of well-known metrics of this type are Precision, Recall and Receiver Operator Characteristic (ROC) and the area under the ROC curve (Cacheda, Carneiro, Fernández, \& Formoso, 2011). The Rank accuracy measures the ability of the system to sort the recommended items like the user would have done.

\subsection{Related Work}

This section briefly outlines the surveys concerning Recommender Systems. We classify the existing surveys into four categories. The first category deals with general introduction to Recommender Systems research. This includes the works of (Adomavicius \& Tuzhilin, 2005; Bouraga, Jureta, Faulkner, \& Herssens, 2014; Park, Choi, Kim, \& Kim, 2011). The second category of the surveys provide methods; Context-aware systems (Baldauf, Dustdar, \& Rosenberg, 2007), approaches and limitations (M. Sharma, 2013), Collaborative Filtering based on social networks (Yang, Guo, Liu, \& Steck, 2013); Basic approaches in Recommender Systems (Felfernig et al., 2014). The third category is dedicated to the various applications of Recommender Systems. This include travel package recommendations (Patil \& Kolhe, 2014), tourist guide (Umanets, Ferreira, \& Leite, 2014), radio station hosting (Ignatov, Nikolenko, Abaev, \& Konstantinova, 2014) and the fourth category cover the evaluation of the various Recommender Systems techniques (Hornung et al., 2013; Krohn-Grimberghe, Nanopoulos, \& Schmidt-Thieme, 2010; Shinde \& Potey, 2015).

Most of the work on Collaborative Filtering has mainly focused on the traditional two dimensional user/item problem. Research on Recommender Systems algorithms gained prominence in 2006 with the launch of the Netflix \$1Million prize contest to improve the state of movie recommendation. This competition led to much interest among many researchers. Koren et al. have demonstrated that Matrix Factorization models are superior to the classic nearest neighbor techniques (Koren et al., 2009).

The extension of standard Matrix Factorization has incorporated context information such as Time-Aware Matrix Factorization (Liu, Cao, Zhao, \& Yang, 2010), Context-aware Matrix Factorization (Baltrunas, Ludwig, \& Ricci, 2011) and Contextual SLIM (Zheng, Mobasher, \& Burke, 2014). The application of Tensor Factorization (Karatzoglou et al., 2010) and (Ricci, De Gemmis, \& Semeraro, 2012). A detailed survey by (Frolov \& Oseledets, 2016) provides Tensor Factorization methods.

This survey will complement and extend the information on previous Context-aware Collaborative Filtering Recommender Systems surveys. In particular, build on the works of (Bokde, Girase, \& Mukhopadhyay, 2015; Karydi \& Margaritis, 2014; Ricci et al., 2012; Shi, Larson, \& Hanjalic, 2014; Su \& Khoshgoftaar, 2009). The survey by $\mathrm{Su}$ and Khoshgoftaar studied Collaborative Filtering techniques and provided a comprehensive coverage of the memory, model and hybrid based approaches outlining the merits, demerits and challenges. Karydi and Margaritis survey presents the use of parallel and distributed systems in the field of Recommender Systems. The survey provides a detailed background to Recommender Systems that employ parallel and/or distributed techniques. Ricci et al. present in their works a survey of Matrix and Tensor Factorization while 
Bokde et al. studied Matrix Factorization models and how they are used in Collaborative Filtering.

\subsection{Organization}

The rest of the paper consists of four sections. The following section describes the methodology used in this research while section 3 outlines Context-aware Matrix Factorization techniques. The conclusion and some suggestions for future directions are given in the last section.

\section{Methodology}

We conducted a focused literature review to identify research papers on Context-aware Matrix Factorization techniques focusing on the limitations, merits and challenges of existing Context-aware Collaborative Filtering Recommender Systems from 2007 to 2016. The following databases were researched: ACM Digital Library, IEEE Xplore digital library, Google Scholar and online Open Access Journals. The search terms for the Boolean search techniques were Recommender Systems, Collaborative Filtering, Matrix Factorization and their combinations with Context-aware. The duplicates were removed from the papers identified in the initial search. Titles and abstracts of the remaining papers were reviewed for relevance to the survey and those that did not meet our requirements were removed. Finally, a full text review was conducted for the remaining papers on CARS based on Matrix Factorization techniques.

\section{Matrix Factorization Techniques for Context-aware Collaborative Filtering Recommender Systems}

\subsection{Context-aware Collaborative Filtering}

The concept of Context-awareness in software applications systems has been conversed by research communities in many different application domains. Thus various definitions of context exists in all the disciplines in which it has been studied, including ubiquitous and pervasive computing, mobile computing, e-commerce, information retrieval, marketing and management as well as in several engineering disciplines. Therefore, the precise definition of what is context varies depending on the Recommender Systems application domain (Bazire \& Brézillon, 2005). We adopt a widely accepted definition of context as any information that can be used to characterize the situation of an entity (Dey \& Abowd, 2000). An entity is a person, place, or object that is considered relevant to the interaction between a user and an application, including the user and applications themselves. Context usually plays the role of additional information besides users, items and the ratings which may be relevant at the current time to make a recommendation. The aim of incorporating contextual information is to generate more relevant suggestions by adapting them to the user's contextual situation.

Context-aware systems sense and adapt their behavior based on the changing contexts (Coutaz, Crowley, Dobson, \& Garlan, 2005), which generally consists of four basic components: Context Acquisition, Context Discovery, Context Model, and Context Processing (Baldauf et al., 2007). The traditional two dimensional Recommender Systems deal with two types of entities, users and items and try to estimate unknown ratings in the Users $\times$ Items matrix.

In the past decade, a number of Recommender Systems algorithms and applications have been developed that incorporate contextual information into the recommendation algorithms. Sarkaleh et al. (Sarkaleh, Mahdavi, \& Baniardalan, 2012) proposed a model which is able to recommend new locations to visitors in a museum while the visitor is given essential information about the certain features of the suggested site that puts into consideration the special needs of students, tourists and the ordinary people. The feature based personalization recommendation system categorized under knowledge level, instruments, ethnic language and tools considered the visitors use to acquire the contents of artistic works. In TripAdvisor (Wang, Chan, \& Ngai, 2012), an application focusing on the attractions on offer, incorporated demographic data to investigate the applicability in recommender algorithms integrated with machine learning methods for the prediction of ratings of tourist attractions. The role of emotions as context variable (Zheng, Mobasher, \& Burke, 2013) evaluated two types of popular Context-aware recommendation algorithms - Context-aware splitting approaches and differential context modelling. The results showed that emotion-linked context makes an important contribution to Context-aware recommendations. The use of additional contextual information data such as weather, time, social media sentiment and user preferences can provide a more accurate model of the user's current context resulting in improved recommendations (Meehan, Lunney, Curran, \& McCaughey, 2013).

\subsection{Matrix Factorization Techniques}

Collaborative Filtering suffers from data sparsity and scalability problem as the number of users and items grow. Matrix decomposition has emerged as a powerful tool to expose hidden structure behind the data. Some of the commonly used Matrix Factorization models are Singular Value Decomposition (Sarwar, Karypis, Konstan, \& Riedl, 2000), Principal Component Analysis (PCA) (Goldberg \& Roeder, 2014), Probabilistic Matrix 
Factorization (PMF) (Salakhutdinov \& Mnih, 2008) and Non-Negative Matrix Factorization (Cai, He, Han, \& Huang, 2011). The matrix decomposition methods are based on Matrix Factorization (Koren et al., 2009; Rendle et al., 2011; Sarwar et al., 2000; Takács et al., 2008). Their results showed the SVD based prediction algorithms can overcome the sparsity problem by utilizing the latent relationships. SVD could be further improved by adding biases to users and items. Koren et al. proposed SVD++ to make use of implicit feedback, reported high accuracy at the expense of high computational cost.

Tensor Factorization (TF) extends the traditional two-dimensional Matrix Factorization problem into an n-dimensional version of the same problem by incorporating contextual information (Gautam, Chaudhary, Sindhwani, \& Bedi, 2016). The resulting multi-dimensional matrix is factored into lower-dimensional representation, where the user, the item and each contextual dimension are represented with a lower dimensional feature vector (Baltrunas et al., 2011; Hidasi \& Tikk, 2013). Karatzoglou et al. (Karatzoglou et al., 2010) proposed a multiverse recommendation model by employing CF method based on Tensor Factorization.

Some studies focus on interpretation of latent features that result from factorization of the user-item matrix. The features extracted from the Non-negative Matrix Factorization are used to establish relationships between users and items (Zhang, Wang, Ford, \& Makedon, 2006). In these works, features are viewed as groups of users, groups or items or as attributes of items. These interpretations require human intervention. In (Brun, Aleksandrova, \& Boyer, 2014) latent features are interpreted as users.

\subsection{Context-aware Matrix Factorization}

There are three basic approaches to develop Context-aware recommendation algorithms as described in (Adomavicius \& Mobasher, 2011): pre-filtering, post-filtering, and contextual modeling. In pre-filtering approach, contextual information is used to filter out irrelevant ratings before they are used for computing recommendations. Three approaches exists in Context-aware splitting approaches (CASA): User, Item and User-Item splitting. In Item splitting (Baltrunas \& Ricci, 2014); (Baltrunas \& Ricci, 2009), multiple copies of an item are created to hold ratings generated in different context based on the contexts in which the rating has been rated. When a recommendation is sought, only those items matching the current context are considered. In the case of User splitting (Baltrunas \& Amatriain, 2009); (Said, De Luca, \& Albayrak, 2011), which splits Users instead of Items. The third CASA is the User-Item splitting, a combination of item splitting and user splitting. The User-Item splitting approach has been shown to be superior to the separate Item or User splitting approaches (Zheng, Mobasher, et al., 2013).

In post-filtering approach, contextual information is used after the classical two dimensional recommendation methods are applied to the non-contextual recommendation data (Baltrunas \& Ricci, 2014); (Zheng, Burke, \& Mobasher, 2014);(Zheng, Mobasher, \& Burke, 2015). Two approaches are common in post-filtering: model-based and heuristic. In model-based, items are filtered out from the recommended result list by building models to predict the probability that the recommended item is relevant to the user in a given contextual situation. Items that have a lower probability than a set threshold are filtered from the list and re-ranking is done based on the probability -weighted rating.

Contextual modeling consists of using the context within the recommendations models (Zheng, Burke, \& Mobasher, 2012); (Zheng, Burke, \& Mobasher, 2013). Contextual modeling incorporates contextual information directly into its recommendation process. Predictive models like Context-aware matrix factorizations, regression and decision trees are examples of contextual modeling techniques that incorporate context into their approach.

The social network approach to provide recommendations for a user based on the ratings of the users that have direct or indirect social relations with the given user. Trust-based Context-aware Matrix Factorization (TCMF) incorporate trust information into both user bias and user-item-context interaction (Li, Sun, \& Lv, 2014). The factorization method builds on the works of (Jamali \& Ester, 2010) on Trust-Aware Collaborative Filtering. In (Li, Yang, \& Jiang, 2016), the static trust model is extended through the use of the Social Network Analysis into new Dynamic Trust-based Context-aware Matrix Factorization (DTCMF) to fully capture the dynamics of trust. The comparative results shows superior performance by the dynamic trust Context-aware Matrix Factorization techniques.

Comparative study of Boolean Matrix Factorization (BMF) with SVD is presented by (Akhmatnurov \& Ignatov, 2015). The experiments with the Boolean matrix product of binary matrices with and without incorporating contextual information with SVD reported a higher precision for BMF where the number of neighbors is not high.

A Tensor Factorization model based on fuzzy mapping between context factors and latent factors is proposed by 
(Fang \& Guo, 2013). In this work movie tags and release time we used as the contextual variables. The combination of tags and release time was modelled as the multidimensional context. The TimeTag (TTSVD) was reported to have achieved better RMSE and HLU while reducing iteration number by $25 \%$.

The Sparse Linear method (SLIM) was designed for Top-N recommendation in the traditional Recommender Systems (Ning \& Karypis, 2011) improves upon the item-based nearest neighbor Collaborative Filtering by learning directly from the data, a sparse matrix of aggregation coefficients that are similar to the traditional item-item similarities. Contextual SLIM (Zheng, Mobasher, et al., 2014), which is derived from the SLIM incorporates contextual information is a Matrix Factorization approach for Top-N recommendations. Table 1 provides a summary of the various works in Context-aware Matrix Factorization techniques, evaluation metrics and the results of the comparative studies.

Table 1. Summary of Context-aware Matrix Factorization techniques

\begin{tabular}{|c|c|c|c|c|}
\hline Reference & MF Technique & Description & Metrics & Results \\
\hline (Li et al., 2016) & $\begin{array}{l}\text { Dynamic } \\
\text { Trust-based } \\
\text { Context-aware } \\
\text { Matrix } \\
\text { Factorization for } \\
\text { Collaborative } \\
\text { Filtering } \\
\text { (DTCMF). } \\
\end{array}$ & $\begin{array}{l}\text { Uses social network analysis to create dynamic } \\
\text { trust model with context- aware matrix } \\
\text { factorization. DTCM-I (both user bias and item } \\
\text { bias are static) while } \\
\text { DTCMF-II (user bias is } \\
\text { con-text-trust-changing and item bias is } \\
\text { context-changing.) }\end{array}$ & MAE & $\begin{array}{l}\text { The DTCMF-I \& II both } \\
\text { outperforms TACF, } \\
\text { TCMF-I\&II }\end{array}$ \\
\hline (Li et al., 2014) & $\begin{array}{l}\text { Incorporating } \\
\text { context into the } \\
\text { Trust-Aware } \\
\text { Collaborative } \\
\text { Filtering (TACF). } \\
\text { Trust-based } \\
\text { Context-aware } \\
\text { Matrix } \\
\text { Factorization for } \\
\text { Collaborative } \\
\text { Filtering (TCMF-I } \\
\text { \& TCMF-II). }\end{array}$ & $\begin{array}{l}\text { Two approaches to incorporate contextual } \\
\text { information into TACF. Incorporates trust } \\
\text { information into both user bias and } \\
\text { user-item-context interaction. TCMF-I (both } \\
\text { user bias and item bias are static while) while } \\
\text { in TCMF-II (user bias is con- } \\
\text { text-trust-sensitive and item bias is } \\
\text { context-sensitive). }\end{array}$ & MAE & $\begin{array}{l}\text { The TCMF-I \& II } \\
\text { outperforms ICAMF-I \& } \\
\text { II models. }\end{array}$ \\
\hline $\begin{array}{l}\text { (Akhmatnurov \& } \\
\text { Ignatov, 2015) }\end{array}$ & $\begin{array}{l}\text { Boolean Matrix } \\
\text { Factorisation } \\
(\mathrm{BMF})\end{array}$ & $\begin{array}{l}\text { Comparative study BMF with SVD. } \\
\text { Experiments on decomposition of the original } \\
\text { matrix into a Boolean matrix product of binary } \\
\text { matrices with/without incorporating contextual } \\
\text { information comparing with SVD. }\end{array}$ & $\begin{array}{l}\text { MAE, Precision } \\
\text { and Recall, } \\
\text { F-measure. }\end{array}$ & $\begin{array}{l}\text { Higher precision } \\
\text { reported for BMF. }\end{array}$ \\
\hline $\begin{array}{l}\text { (Fang \& Guo, } \\
2013)\end{array}$ & $\begin{array}{l}\text { TagSVD (T-SVD) } \\
\text { and TimeTagSVD } \\
\text { (TT-SVD). }\end{array}$ & $\begin{array}{l}\text { TF model based on fuzzy mapping between } \\
\text { context factors and latent factors. Improved the } \\
\text { performance of SVD, bias SVD and BPMF } \\
\text { using SGD and MCMC. }\end{array}$ & $\begin{array}{l}\text { MAE, RMSE } \\
\text { Half Life Utility } \\
\text { (HLU) }\end{array}$ & $\begin{array}{l}\text { TT-SVD outperforms } \\
\text { SVD, NMF \& Slope } \\
\text { One. }\end{array}$ \\
\hline $\begin{array}{l}\text { (Karatzoglou et } \\
\text { al., 2010) }\end{array}$ & $\begin{array}{l}\text { Multiverse Tensor } \\
\text { Factorization } \\
\text { recommendation } \\
\text { model based on CF. }\end{array}$ & $\begin{array}{l}\text { Tensor Factorization allowed integration of } \\
\text { contextual information resulting in a } \\
\text { multidimensional matrix of User-Item Context } \\
\text { as a tensor, where context can be taken in any } \\
\text { number. }\end{array}$ & $\mathrm{M}$ & $\begin{array}{l}\text { TF outperforms both } \\
\text { contextual pre-filtering } \\
\text { methods and the context } \\
\text { free MF }\end{array}$ \\
\hline $\begin{array}{l}\text { (Zheng et al., } \\
\text { 2015) }\end{array}$ & $\begin{array}{l}\text { Context } \\
\text { Correlation-based } \\
\text { Context-aware } \\
\text { Matrix } \\
\text { Factorization } \\
\text { (Correlation-based } \\
\text { CAMF). } \\
\end{array}$ & $\begin{array}{l}\text { Used the correlations between contextual } \\
\text { deviations and incorporated into the Matrix } \\
\text { Factorization to formulate the } \\
\text { correlation-based CAMF algorithm. }\end{array}$ & $\begin{array}{l}\text { Precision, Mean } \\
\text { Average } \\
\text { Precision (MAP) }\end{array}$ & $\begin{array}{l}\text { Correlation-based } \\
\text { CAMF outperforms } \\
\text { deviation-based CAMF } \\
\text { and the TF algorithm. }\end{array}$ \\
\hline $\begin{array}{l}\text { (Zheng, } \\
\text { Mobasher, et al., } \\
\text { 2014) }\end{array}$ & $\begin{array}{lr}\text { Contextual } & \text { Sparse } \\
\text { Linear } & \text { Method } \\
\text { (CSLIM). } & \\
\end{array}$ & $\begin{array}{l}\text { MF approach for Top-N recommendations that } \\
\text { incorporates contextual information. }\end{array}$ & $\begin{array}{l}\text { Precision and } \\
\text { MAP }\end{array}$ & $\begin{array}{l}\text { CSLIM outperforms TF, } \\
\text { CAMF and CASA. }\end{array}$ \\
\hline $\begin{array}{ll}\text { (Baltrunas } & \& \\
\text { Ricci, 2014) } & \end{array}$ & $\begin{array}{l}\text { Context dependent } \\
\text { CF using item } \\
\text { splitting. }\end{array}$ & $\begin{array}{l}\text { Used both gradient descent Matrix } \\
\begin{array}{l}\text { Factorization and nearest neighbor CF } \\
\text { algorithms. }\end{array}\end{array}$ & $\begin{array}{l}\text { MAE, Precision } \\
\text { and Recall. }\end{array}$ & $\begin{array}{lr}\text { Item } & \text { splitting with } \\
\text { Matrix } & \text { Factorization } \\
\text { outperforms kNN. }\end{array}$ \\
\hline $\begin{array}{l}\text { (Chen, Zheng, } \\
\text { Wang, Hong, \& } \\
\text { Lin, 2014) }\end{array}$ & $\begin{array}{l}\text { Context-based } \\
\text { Collaborative Topic } \\
\text { Regression Social }\end{array}$ & $\begin{array}{l}\text { Use spectral clustering for user-item } \\
\text { subgroupings, so as to group users and items in } \\
\text { similar context. Incorporate topic modelling. }\end{array}$ & $\begin{array}{lr}\text { MAE, } & \text { Recall } \\
\text { and Root } & \text { Mean } \\
\text { Square } & \text { Error } \\
\end{array}$ & $\begin{array}{l}\text { C-CTR-SMF2 } \\
\text { outperforms PMF, SoCo, } \\
\text { fLDA, CTR and }\end{array}$ \\
\hline
\end{tabular}




\begin{tabular}{|c|c|c|c|c|}
\hline Reference & MF Technique & Description & Metrics & Results \\
\hline & $\begin{array}{l}\text { Matrix } \\
\text { Factorization } \\
\text { (C-CTR-SMF2). }\end{array}$ & $\begin{array}{l}\text { Use hierarchical Bayesian model to make } \\
\text { predictions for each user-item subgroup. }\end{array}$ & (RMSE). & CTR-SMF. \\
\hline $\begin{array}{l}\text { (Li, Feng, \& Lv, } \\
\text { 2013) }\end{array}$ & $\begin{array}{l}\text { Improved CAMF: } \\
\text { ICAMF-I }\end{array}$ & $\begin{array}{l}\text { Both user bias and item bias are static and } \\
\text { ICAMF-II (the timely change of user bias and } \\
\text { item bias over different context.) }\end{array}$ & MAE & $\begin{array}{l}\text { Both ICAMF-I\&II } \\
\text { outperforms CAMF-C, } \\
\text { CAMF-CC and } \\
\text { CAMF-CI models. }\end{array}$ \\
\hline $\begin{array}{l}\text { (Baltrunas et al., } \\
\text { 2011) }\end{array}$ & $\begin{array}{l}\text { Dealt with varying } \\
\text { granularities of the } \\
\text { interaction of the } \\
\text { contextual } \\
\text { information with } \\
\text { ratings. CAMF-C, } \\
\text { CAMF-CI and } \\
\text { CAMF-CC }\end{array}$ & $\begin{array}{l}\text { In CAMF-C every contextual factor had a } \\
\text { global influence on the ratings independently } \\
\text { from the item. CAMF-CI introduced one } \\
\text { parameter per contextual factor and item pair. } \\
\text { One parameter was introduced for each item } \\
\text { category and contextual factor in CAMF-CC. }\end{array}$ & MAE & $\begin{array}{l}\text { CAMF-CC outperforms } \\
\text { CAMF, CAMF-C and } \\
\text { CAMF-CI. }\end{array}$ \\
\hline (Cai et al., 2011) & $\begin{array}{l}\text { Graph regularized } \\
\text { Non-Negative } \\
\text { Matrix } \\
\text { Factorization } \\
\text { (GNMF) for data } \\
\text { representation. }\end{array}$ & $\begin{array}{l}\text { Construct an affinity graph to encode the } \\
\text { geometrical information of the data space by } \\
\text { constructing a nearest neighbor graph. }\end{array}$ & $\begin{array}{l}\text { Accuracy (AC) } \\
\text { and Normalized } \\
\text { Mutual } \\
\text { Information } \\
\text { (NMI) }\end{array}$ & outperforms \\
\hline
\end{tabular}

\section{Conclusion and Future Work}

This work provided a broad overview of available approaches to incorporating contextual information into Collaborative Filtering based Recommender Systems utilizing Matrix Factorization techniques. Although the field of Recommender Systems has developed considerably, the aspects of quality of recommendations, sparsity, scalability, cold start and privacy concerns have remained unresolved since the beginning of Recommender Systems research. Recommender Systems whatever their approach in providing recommendations has proven to be useful in overcoming the problem of information overload by delivering relevant personalized information. $\mathrm{CF}$ is the most successful and widely used recommendation technique. However, just like the other approaches, the technique suffers from limitations of data sparsity and scalability problems that hamper the performance, quality and the accuracy of the predictions. The main reason behind data sparsity in Collaborative Filtering is that most users do not rate most of the items making the available ratings sparse. CF suffers from this problem because it is dependent on the rating matrix. The incorporation of contextual information has been suggested as a remedy to overcome the traditional Recommender Systems problems. Context plays the role of additional information besides users, items and the ratings which may be relevant at the current time to make a recommendation.

Matrix decomposition is a powerful technique to find the hidden structure behind the data. SVD, Non-negative Matrix Factorization and Probabilistic Matrix Factorization are popular decomposition models. SVD is able to handle large dataset, sparseness of rating matrix and scalability problem of CF algorithm efficiently. NMF is widely used to reduce dimensions and extract latent factors. PMF models place Gaussian priors on user and movie features, and turn the recommendation task into a probabilistic problem. Matrix/Tensor Factorization models could be optimized by using Stochastic Gradient Descent (SGD), Alternating Least Squares (ALS), or Markov Chain Monte Carlo Inference (MCMC). Tensor Factorization models are not popular in practical application for its high computational cost. The various available evaluation metrics can also be applied to evaluate performance of Recommender Systems by measuring their coverage and accuracy, but the current metrics are insufficient for evaluating quality and usefulness of Recommender Systems. Recommender Systems evaluation metrics are trapped in the recommendation accuracy.

Future research directions of the CARS should be directed towards a unified definition of context and what constitutes contextual information. Another important aspect to consider is to address the privacy concerns that can be generated by the incorporation of contextual information. The conduct of more research in user studies is critical to obtain the necessary feedback and to get out of the accuracy cage. Although many advanced techniques have been developed to address data sparsity, it still remains a growing problem that needs to be addressed. The need for fast and scalable computations is critical and therefore great effort must be expended to develop efficient and scalable algorithms.

\section{References}

Adomavicius, G., \& Mobasher, B. (2011). Context-aware Recommender Systems. Artificial Intelligence 
Magazine, 32(3), 67-80.

Adomavicius, G., \& Tuzhilin, A. (2005). Toward the Next Generation of Recommender Systems: A survey of the State-of-the-Art and Possible Extensions. IEEE Transactions on Knowledge and Data Engineering, 17(6), 734-749. https://doi.org/10.1109/TKDE.2005.99

Adomavicius, G., Sankaranarayanan, R., Sen, S., \& Tuzhilin, A. (2005). Incorporating Contextual Information in Recommender Systems Using a Multidimensional Approach. ACM Transactions on Information Systems, 23(1), 103-145. https://doi.org/10.1145/1055709.1055714

Akhmatnurov, M., \& Ignatov, D. I. (2015). Context-aware recommender system based on Boolean matrix factorisation. In CEUR Workshop Proceedings (Vol. 1466, pp. 99-110).

Baldauf, M., Dustdar, S., \& Rosenberg, F. (2007). A survey on Context-aware systems. International Journal Ad Hoc and Ubiquitous Computing, 2(4), 263-277. https://doi.org/10.1504/IJAHUC.2007.014070

Baltrunas, L. (2008). Exploiting contextual information in recommender systems. In Proceedings of the 2008 ACM conference on Recommender systems - RecSys '08 (p. 295). https://doi.org/10.1145/1454008.1454056

Baltrunas, L., \& Amatriain, X. (2009). Towards time-dependant recommendation based on implicit feedback. Workshop on Context-Aware Recommender Systems (CARS'09), 25-30. https://doi.org/10.1109/ICDM.2013.139

Baltrunas, L., \& Ricci, F. (2009). Context-based splitting of item ratings in Collaborative Filtering. Proceedings of the Third ACM Conference on Recommender Systems - RecSys '09, 245. https://doi.org/10.1145/1639714.1639759

Baltrunas, L., \& Ricci, F. (2014). Experimental evaluation of context-dependent Collaborative Filtering using item splitting. User Modeling and User-Adapted Interaction, 24(1-2), 7-34. https://doi.org/10.1007/s11257-012-9137-9

Baltrunas, L., Ludwig, B., \& Ricci, F. (2011). Matrix Factorization Techniques for Context aware recommendation. In Acm Rs (pp. 301-304). https://doi.org/10.1145/2043932.2043988

Bazire, M., \& Brézillon, P. (2005). Understanding context before using it. In 5th International and Interdisciplinary Conference (Vol. 3554, pp. 29-40). https://doi.org/10.1007/11508373

Bokde, D., Girase, S., \& Mukhopadhyay, D. (2015). Matrix Factorization model in Collaborative Filtering algorithms: A survey. Procedia Computer Science, 49(1), 136-146. https://doi.org/10.1016/j.procs.2015.04.237

Bouraga, S., Jureta, I., Faulkner, S., \& Herssens, C. (2014). Knowledge-Based Recommendation Systems: International Journal of Intelligent Information Technologies, 10(2), 1-19. https://doi.org/10.4018/ijiit.2014040101

Breese, J. S., Heckerman, D., \& Kadie, C. (1998). Empirical analysis of predictive algorithms for Collaborative Filtering. In UAI'98 Proceedings of the Fourteenth Conference on Uncertainty in Artificial Intelligence (pp. 43-52). https://doi.org/10.1111/j.1553-2712.2011.01172.x

Brun, A., Aleksandrova, M., \& Boyer, A. (2014). Can latent features be interpreted as users in matrix factorization-based recommender systems? In Proceedings - 2014 IEEE/WIC/ACM International Joint Conference on Web Intelligence and Intelligent Agent Technology - Workshops, WI-IAT 2014 (Vol. 2, pp. 257-297). https://doi.org/10.1109/WI-IAT.2014.102

Cacheda, F., Carneiro, V., Fernández, D., \& Formoso, V. (2011). Comparison of Collaborative Filtering algorithms: Limitations of Current Techniques and Proposals for Scalable, High-Performance Recommender Systems. ACM Transactions on the Web, 5(1), 1-33. https://doi.org/10.1145/1921591.1921593

Cai, D., He, X., Han, J., \& Huang, T. S. (2011). Graph regularized Non-negative Matrix Factorization for data representation. IEEE Transactions on Pattern Analysis and Machine Intelligence, 33(8), 1548-1560. https://doi.org/10.1007/978-3-642-34528-9_1

Chen, C., Zheng, X., Wang, Y., Hong, F., \& Lin, Z. (2014). Context-aware Collaborative Topic Regression with Social Matrix Factorization for Recommender Systems. In Twenty-Eighth AAAI Conference (pp. 9-15).

Chertov, O., Brun, A., Boyer, A., \& Aleksandrova, M. (2015). Comparative analysis of neighborhood-based approache and Matrix Factorization in recommender systems. Eastern-European Journal of Enterprise 
Technologies, 3(4(75)), 4. https://doi.org/10.15587/1729-4061.2015.43074

Coutaz, J., Crowley, J. L., Dobson, S., \& Garlan, D. (2005). Context is key. Communications of the ACM, 48(3), 49. https://doi.org/10.1145/1047671.1047703

Dey, A. K., \& Abowd, G. D. (2000). Towards a better understanding of context and Context-awareness. CHI 2000 Workshop on the What Who Where When and How of Context-awareness, 4(What, Who, Where, When and How of Context-Awareness), 1-6. https://doi.org/10.1007/3-540-48157-5

Fang, Y., \& Guo, Y. (2013). A Context-aware Matrix Factorization recommender algorithm. In Proceedings of the IEEE International Conference on Software Engineering and Service Sciences, ICSESS (pp. 914-918). https://doi.org/10.1109/ICSESS.2013.6615454

Felfernig, A., Jeran, M., Ninaus, G., Reinfrank, F., Reiterer, S., \& Stettinger, M. (2014). Basic approaches in recommendation systems. Recommendation Systems in Software Engineering, 15-37. https://doi.org/10.1007/978-3-642-45135-5_2

Frolov, E., \& Oseledets, I. (2016). Tensor methods and recommender systems. Wiley Interdisciplinary Reviews: Data Mining and Knowledge Discovery, 7(3), 1-41. https://doi.org/10.1002/widm.1201

Gao, M., Liu, K., \& Wu, Z. (2010). Personalisation in web computing and informatics: Theories, techniques, applications, and future research. Information Systems Frontiers, 12(5), 607-629. https://doi.org/10.1007/s10796-009-9199-3

Gautam, A., Chaudhary, P., Sindhwani, K., \& Bedi, P. (2016). CBCARS: Content boosted Context-aware recommendations using tensor factorization. 2016 International Conference on Advances in Computing, Communications and Informatics, ICACCI 2016, 75-81. https://doi.org/10.1109/ICACCI.2016.7732028

Goldberg, K., \& Roeder, T. M. (2014). Eigentaste: A Constant Time Collaborative Filtering Algorithm. CEUR Workshop Proceedings, 1225(July), 41-42. https://doi.org/10.1023/A

Herlocker, J. L., Konstan, J. A., Terveen, L. G., \& Riedl, J. T. (2004). Evaluating Collaborative Filtering recommender systems. ACM Transactions on Information Systems, 22(1), 5-53. https://doi.org/10.1145/963770.963772

Hidasi, B., \& Tikk, D. (2013). Context-aware item-to-item recommendation within the factorization framework. In Proceedings of the 3rd Workshop on Context-awareness in Retrieval and Recommendation - CaRR '13 (pp. 19-25). https://doi.org/10.1145/2442670.2442675

Hornung, T., Ziegler, C. N., Franz, S., Przyjaciel-Zablocki, M., Schätzle, A., \& Lausen, G. (2013). Evaluating hybrid music recommender systems. Proceedings - 2013 IEEE/WIC/ACM International Conference on Web Intelligence, WI 2013, 1, 57-64. https://doi.org/10.1109/WI-IAT.2013.9

Ignatov, D. I., Nikolenko, S., Abaev, T., \& Konstantinova, N. (2014). Online Recommender System for Radio Station Hosting: Experimental Results Revisited. In 2014 IEEE/WIC/ACM International Joint Conferences on Web Intelligence (WI) and Intelligent Agent Technologies (IAT) (pp. 229-236). https://doi.org/10.1109/WI-IAT.2014.38

Jamali, M., \& Ester, M. (2010). A Matrix Factorization technique with trust propagation for recommendation in social networks. In Proceedings of the fourth ACM conference on Recommender systems - RecSys '10 (p. 135). https://doi.org/10.1145/1864708.1864736

Karatzoglou, A., Amatriain, X., Baltrunas, L., \& Oliver, N. (2010). Multiverse recommendation: n-dimensional Tensor Factorization for Context-aware Collaborative Filtering. Proceedings of the Fourth ACM Conference on Recommender Systems - RecSys '10, 79. https://doi.org/10.1145/1864708.1864727

Karydi, E., \& Margaritis, K. (2014). Parallel and Distributed Collaborative Filtering: A Survey. arXiv Preprint arXiv:1409.2762, 49(2). https://doi.org/10.1145/2951952

Koren, Y., \& Bell, R. (2015). Advances in Collaborative Filtering. Recommender Systems Handbook, Second Edition, 77-118. https://doi.org/10.1007/978-1-4899-7637-6_3

Koren, Y., Bell, R., \& Volinsky, C. (2009). Matrix factorization techniques for recommender systems. Computer, 42(8), 30-37. https://doi.org/10.1109/MC.2009.263

Krohn-Grimberghe, A., Nanopoulos, A., \& Schmidt-Thieme, L. (2010). A novel multidimensional framework for evaluating recommender systems. CEUR Workshop Proceedings, 612, 34-41.

Li, J., Feng, P., \& Lv, J. (2013). ICAMF: Improved Context-aware Matrix Factorization for Collaborative 
Filtering. Proceedings - International Conference on Tools with Artificial Intelligence, ICTAI, 63-70. https://doi.org/10.1109/ICTAI.2013.20

Li, J., Sun, C., \& Lv, J. (2014). TCMF:Trust-Based Context-Aware Matrix Factorization for Collaborative Filtering. In 2014 IEEE 26th International Conference on Tools with Artificial Intelligence (pp. 815-821). https://doi.org/10.1109/ICTAI.2014.126

Li, J., Yang, R., \& Jiang, L. (2016). DTCMF: Dynamic trust-based Context-aware Matrix Factorization for Collaborative Filtering. In Proceedings of 2016 IEEE Information Technology, Networking, Electronic and Automation Control Conference, ITNEC 2016 (pp. 914-919). https://doi.org/10.1109/ITNEC.2016.7560495

Liu, N. N., Cao, B., Zhao, M., \& Yang, Q. (2010). Adapting neighborhood and Matrix Factorization models for context aware recommendation. In CAMRa2010 (pp. 7-13). https://doi.org/10.1145/1869652.1869653

Meehan, K., Lunney, T., Curran, K., \& McCaughey, A. (2013). Context-aware intelligent recommendation system for tourism. 2013 IEEE International Conference on Pervasive Computing and Communications Workshops, PerCom Workshops 2013, (March), 328-331. https://doi.org/10.1109/PerComW.2013.6529508

Melville, P., Mooney, R. J., \& Nagarajan, R. (2002). Content-boosted Collaborative Filtering for improved recommendations. "Proceedings of the 18th National Conference on Artificial Intelligence (AAAI), " (July), 187-192. https://doi.org/10.1.1.16.4936

Nguyen, J., \& Zhu, M. (2013). Content-boosted Matrix Factorization techniques for recommender systems. Statistical Analysis and Data Mining, 6(4), 286-301. https://doi.org/10.1002/sam.11184

Ning, X., \& Karypis, G. (2011). SLIM: Sparse LInear Methods for top-N recommender systems. In Proceedings - IEEE International Conference on Data Mining, ICDM (pp. 497-506). https://doi.org/10.1109/ICDM.2011.134

Park, D. H., Choi, I. Y., Kim, H. K., \& Kim, J. K. (2011). A Review and Classification of Recommender Systems Research. 2011 International Conference on Social Science and Humanity, 5(1), $290-294$. https://doi.org/10.7763/IPEDR

Patil, P., \& Kolhe, V. L. (2014). Survey of Travel Package Recommendation System. International Journal of Science and Research, 3(12), 1557-1561.

Rajabpour, N., Bardsiri, V. K., Mohammadighavam, A., \& Molaei, E. (2014). Application domain of recommender system: a survey. International Journal of Advanced Studies in Computer Science and Engineering, 3(2), 8-14.

Rendle, S., Gantner, Z., Freudenthaler, C., \& Schmidt-Thieme, L. (2011). Fast Context-aware recommendations with factorization machines. Proceedings of the 34th International ACM SIGIR Conference on Research and Development in Information - SIGIR '11, 635. https://doi.org/10.1145/2009916.2010002

Ricci, G., De Gemmis, M., \& Semeraro, G. (2012). Matrix and Tensor Factorization Techniques applied to Recommender Systems: a Survey. Matrix and Tensor Factorization Techniques Applied to Recommender Systems: A Survey, 1(1), 94-98.

Said, A., De Luca, E. W., \& Albayrak, S. (2011). Inferring contextual user profiles improving recommender performance. CEUR Workshop Proceedings, 791.

Salakhutdinov, R., \& Mnih, A. (2008). Bayesian probabilistic Matrix Factorization using Markov chain Monte Carlo. In Proceedings of the 25th international conference on Machine learning - ICML '08 (Vol. 25, pp. 880-887). https://doi.org/10.1145/1390156.1390267

Sarkaleh, M. K., Mahdavi, M., \& Baniardalan, M. (2012). Designing a Tourism Recommender System Based on Location, Mobile Device and User Features in Museum. International Journal of Managing Information Technology, 4(2), 13-21. https://doi.org/10.5121/ijmit.2012.4202

Sarwar, B. M., Karypis, G., Konstan, J. a, \& Riedl, J. T. (2000). Application of Dimensionality Reduction in Recommender System - A Case Study. Architecture, 1625, 264-8. https://doi.org/10.1.1.38.744

Sharma, L., \& Gera, A. (2013). A Survey of Recommendation System: Research Challenges. International Journal of Engineering Trends and Technology, 4(5), 1989-1992.

Sharma, M. (2013). A Survey of Recommender Systems: Approaches and Limitations. International Journal of Innovations in Engineering and Technology, 1-9.

Shi, Y., Larson, M., \& Hanjalic, A. (2014). Collaborative Filtering beyond the User-Item Matrix: A Survey of the 
State of the Art and Future Challenges. ACM Computing Surveys (CSUR), 47(1), 1-45. https://doi.org/10.1145/2556270

Shinde, S., \& Potey, M. A. (2015). Survey on Evaluation of Recommender Systems. International Journal of Engineering And Computer Science ISSN:2319-7242, 4(2), 1-5.

$\mathrm{Su}, \mathrm{X}$., \& Khoshgoftaar, T. M. (2009). A Survey of Collaborative Filtering Techniques. Advances in Artificial Intelligence, 2009(Section 3), 1-19. https://doi.org/10.1155/2009/421425

Takács, G., Pilászy, I., Németh, B., Tikk, D., Pilaszy, I., N’emeth, B., \& Tikk, D. (2008). Investigation of Various Matrix Factorization Methods for Large Recommender Systems. Proceedings of the 2nd KDD Workshop on Large Scale Recommender Systems and the Netflix Prize Competition, 1, 553-562. https://doi.org/10.1109/ICDMW.2008.86

Umanets, A., Ferreira, A., \& Leite, N. (2014). GuideMe - A Tourist Guide with a Recommender System and Social Interaction. Procedia Technology, 17, 407-414. https://doi.org/10.1016/j.protcy.2014.10.248

Wang, Y., Chan, S. C. F., \& Ngai, G. (2012). Applicability of demographic recommender system to tourist attractions: A case study on TripAdvisor. Proceedings of the 2012 IEEE/WIC/ACM International Conference on Web Intelligence and Intelligent Agent Technology Workshops, WI-IAT 2012, 97-101. https://doi.org/10.1109/WI-IAT.2012.133

Yang, X., Guo, Y., Liu, Y., \& Steck, H. (2013). A survey of Collaborative Filtering based social recommender systems. Computer Communications, (0). https://doi.org/http://dx.doi.org/10.1016/j.comcom.2013.06.009

Zhang, S., Wang, W., Ford, J., \& Makedon, F. (2006). Learning from Incomplete Ratings Using Non-negative Matrix Factorization. In Proceedings of the 2006 SIAM International Conference on Data Mining (pp. 549553). https://doi.org/10.1137/1.9781611972764.58

Zheng, Y., Burke, R., \& Mobasher, B. (2012). Differential context relaxation for Context-aware travel recommendation. Lecture Notes in Business Information Processing, 123 LNBIP, 88-99. https://doi.org/10.1007/978-3-642-32273-0_8

Zheng, Y., Burke, R., \& Mobasher, B. (2013). Recommendation with differential context weighting. Lecture Notes in Computer Science (Including Subseries Lecture Notes in Artificial Intelligence and Lecture Notes in Bioinformatics), 7899 LNCS, 152-164. https://doi.org/10.1007/978-3-642-38844-6_13

Zheng, Y., Burke, R., \& Mobasher, B. (2014). Splitting approaches for Context-aware recommendation: An Empirical Study. In Proceedings of the 29th Annual ACM Symposium on Applied Computing - SAC '14 (pp. 274-279). https://doi.org/10.1145/2554850.2554989

Zheng, Y., Mobasher, B., \& Burke, R. (2014). CSLIM:Contextual SLIM Recommendation Algorithms. In RecSys 2014 - Proceedings of the 8th ACM Conference on Recommender Systems (Vol. 0, pp. 301-304). https://doi.org/10.1145/2645710.2645756

Zheng, Y., Mobasher, B., \& Burke, R. (2015). Incorporating context correlation into Context-aware matrix factorization. In CEUR Workshop Proceedings (Vol. 1440).

Zheng, Y., Mobasher, B., \& Burke, R. D. (2013). The Role of Emotions in Context-aware Recommendation. Decisions@RecSys, 2013,21-28.

\section{Copyrights}

Copyright for this article is retained by the author(s), with first publication rights granted to the journal.

This is an open-access article distributed under the terms and conditions of the Creative Commons Attribution license (http://creativecommons.org/licenses/by/4.0/). 